\section{The use of interstitial echo-guided diode laser 980-nm for deep vascular anomalies in pediatric patients: a preliminary study}

\author{
Luigino Santecchia, ${ }^{1}$ \\ Maria Francesca Bianciardi Valassina, ${ }^{1}$ \\ Guido Ciprandi, ${ }^{1}$ Rodolfo Fruhwirth, ${ }^{2}$ \\ Mario Zama ${ }^{1}$ \\ 'Plastic and Maxillofacial Surgery Unit, \\ Pediatric Hospital Bambino Gesù, IRCCS, \\ Rome; ${ }^{2}$ Vascular and Interventional \\ Radiology Unit, Pediatric Hospital \\ Bambino Gesù, IRCCS, Rome, Italy
}

\section{Abstract}

A wide range of therapeutic options is available to treat vascular anomalies, arising from the systemic therapies to surgery or using lasers. The purpose of this preliminary study is to assess the effectiveness of treatment of vascular anomalies anywhere in the body, along with the use of interstitial echo-guided $980 \mathrm{~nm}$ diode laser. The analysis occurs through accurate angio magnetic resonance imaging (MRI) pre- and post-treatment measurements. We enrolled all the patients (16) affected on vascular malformations everywhere in the body, treated from January to August 2012. We obtained excellent results in 6 patients (37.5\%) with mean mass reduction of $85 \%$, good in 9 patients (56\%) with mean mass reduction of $65 \%$ and unsatisfactory in 1 patient $(6 \%)$. In pediatric patients, low-flow vascular malformations resistant to progressive sclerotherapy or in critical anatomical sites, benefit of echo guided interstitial 980 $\mathrm{nm}$ diode laser.

\section{Introduction}

The infantile hemangiomas (IH) do proliferate during the first year of life from the endothelial cells alone, as alike benign tumors behavior. They usually regress almost in the $80 \%$ of cases within the $7^{\text {th }}$ year of life with no treatment. ${ }^{1,2}$

Vascular malformations otherwise are present at birth in most cases, and do not involve the endothelial proliferation system. They can affect any area of the vascular tree at any level (including arterial, venous, capillary, lymphatic or mixed). The expansion comes up progressively with the body growth, and do not solve spontaneously. ${ }^{3,4}$
A wide range of therapeutic options is available to treat vascular anomalies, arising from the systemic therapies to surgery or using lasers. We might consider some keystones being involved in caring vascular malformations: first, the patient's age and the anatomical location; second, the extent of the lesion and the effectiveness of treatment, which entails both aesthetic as psychological implications; and third, the cost-benefit ratios. ${ }^{5-7}$

The purpose of this preliminary study is to assess the effectiveness of treatment of vascular anomalies anywhere in the body, along with the use of interstitial echo-guided $980 \mathrm{~nm}$ diode laser. The analysis occurs through accurate angio-MRI pre and post treatment measurements.

This preliminary report summarizes a new approach, never been described before in literature, with reference to resistant anomalies to local therapy or dangerous to operate.

\section{Materials and Methods}

In this monocentric study we enrolled all the patients affected on vascular malformations everywhere in the body, treated from January to August 2012, using 980-nm interstitial echoguided diode laser. For each patient the same features were considered: the age at first diagnosis, sex, symptoms, site of the lesion and previous treatments (Table 1 and Figure 1). The patients carried out an MRI to assess 3D sizes and to the same imaging evaluation 3 months after the last laser session.

The treatment was performed under general anesthesia and carried out with cannulas of different sizes needle, according to the lesion range and the anatomic area to be treated.

Under echo-guided visualization with a linear probe (5-7.5 MHz) the insertion of the optical fiber was done according to the Seldinger technique. The cannula came up against the core of the vascular malformation under direct Doppler control. Laser light was set in a power range of 4-10 W (total fluency administered ranging from 800 to $1200 \mathrm{~J}$ ) carring out a continuos mode for all malformations treated for 1 to $3 \mathrm{~min}$, with a frequency ranging from 2 to 4 $\mathrm{Hz}$.

\section{Results}

Sixteen patients in this preliminary study were enrolled. Fifteen presented low-flow vascular malformations and one was a lymphangioma.

The results were fixed mainly evaluating some clinical signs, as visual inspection and cosmetic skin improvement, reduction of
Correspondence: Luigino Santecchia, Plastic and Maxillofacial Surgery Unit, Pediatric Hospital Bambino Gesù, IRCCS, Piazza S. Onofrio 4, 00165 Rome, Italy.

Tel. +39.06.68592365 - Fax: +39.06 .68592201 .

E-mail: luigino.santecchia@opbg.net

Key words: vascular anomalies, low-flow malformation, diode laser.

Contributions: all authors have made substantial contributions to conception and design of the text, the acquisition of data, contributing to the analysis and interpretation of data and drafting of the article with critical revision for important intellectual content, and approved the final version.

Conflict of interests: the authors declare no potential conflict of interests.

Received for publication: 10 May 2013.

Revision received: 10 June 2013.

Accepted for publication: 11 June 2013.

This work is licensed under a Creative Commons Attribution NonCommercial 3.0 License (CC BYNC 3.0).

(C) Copyright L. Santecchia et al., 2013

Licensee PAGEPress, Italy

Surgical Techniques Development 2013; 3:e2 doi:10.4081/std.2013.e2

symptoms (pain, heaviness, sting, pulsatility), functional impairment lessening, and volumetric decrease, through the analysis of pre- and post-treatment MRI values (Figures 2-5).

We obtained excellent results in 6 patients (37.5\%) with mean mass reduction of $85 \%$, good in 9 patients (56\%) with mean mass reduction of $65 \%$, and unsatisfactory in 1 patient $(6 \%)$.

The mean percentage of reduction in our case series was $71 \%$, while for vascular malformations $<10 \mathrm{~cm}$ wide it was $95 \%$.

No dangerous adverse effects were detected. No facial nerve injuries were detected. Patients, however, showed a moderate swelling in the site of application for a few days. The pain was easily controlled with drugs in 2-3 days. No atrophy nor cutaneous hyper and/or hypopigmentation were observed. A little scar was detected in a patient with a cheek vascular malformation. The follow-up period varied from 2 to 4 months.

\section{Discussion}

For a long time vascular anomalies treatment in pediatrics had undergone a continuous refinement, due to difficult grouping and a 
lot of classifications. When a definitive classification was established by the International Society for the Study of Vascular Anomalies (ISVVA) in 1996, the focus shifted to new protocols and less invasive approaches, with minor implication of the patient, both physically as psychologically.

Surgery has partially lost its central role in the management of the Infantile Hemangiomas in favour of local, systemic therapies or lasers in dangerous areas. ${ }^{8-13}$

However, many surgical indications persist, particularly in the presence of ulcerated malformations or deep lesions that can cause hemodynamic instability or in particular

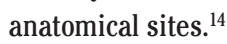

The surgical way often requires some complementary treatments, to maximize the final results in a multidisciplinary approach. ${ }^{15}$

The correct diagnosis and classification of the type of malformation we are facing, do indicate the therapeutic option among a wide range of possibility. ${ }^{16}$ Furthermore, the role of the imaging [echo-color Doppler, vascular-
MRI, multislice computed tomography (CT) and angiography] is crucial for the correct planning.

Some authors ${ }^{17}$ believe that the endolesional diode laser could be reserved to periorbital, intranasal or laryngeal hemangiomas, when we need an extreme selective and less invasive approach to preserve the aesthetic function, or in case we are not capable to arrest bleeding.

We do not agree completely whit this statement, and we do believe some choices move on by several factors and your own personal expe-

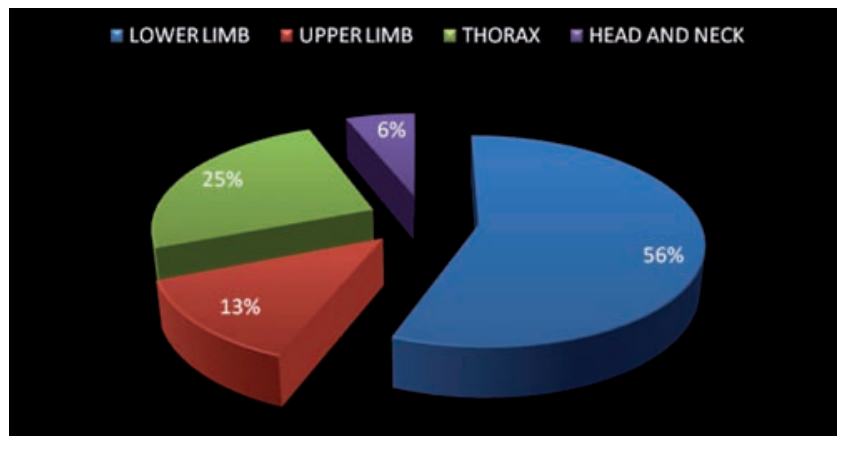

Figure 1. Malformation site distribution of the population studied.

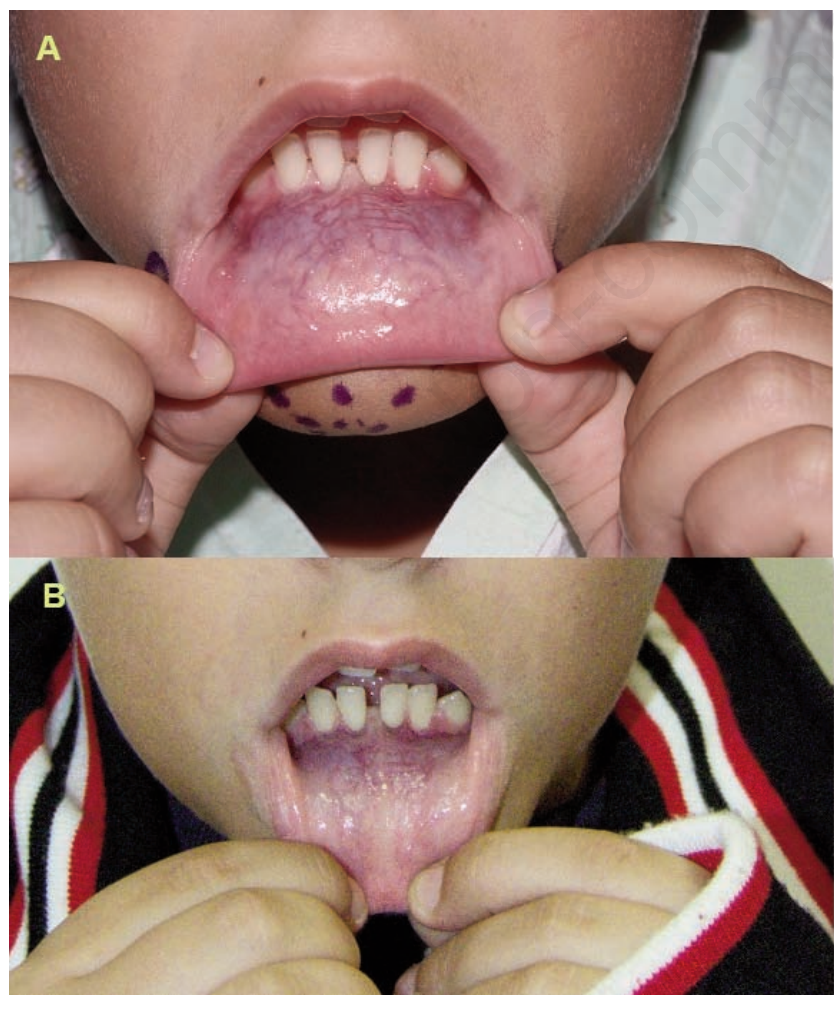

Figure 2. A) Pre-treatment clinical appearance of a chin low flow vascular malformation in a 11-year-old boy, previously treated by sclerotherapy; B) appearence after a single treatment with interstitial echo-guided diode laser 980-nm ( $5 \mathrm{~W}$ for $60 \mathrm{sec}$ ).

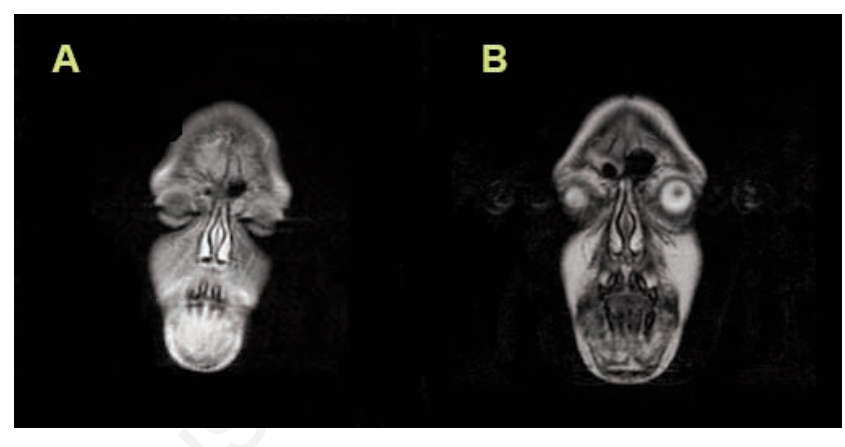

Figure 3. A) Pre-treatment clinical appearance of a chin low flow vascular malformation in a 11-year-old boy, previously treated by sclerotherapy; B) appearence after a single treatment with interstitial echo-guided diode laser $980-\mathrm{nm}$ ( $5 \mathrm{~W}$ for $60 \mathrm{sec}$ ).

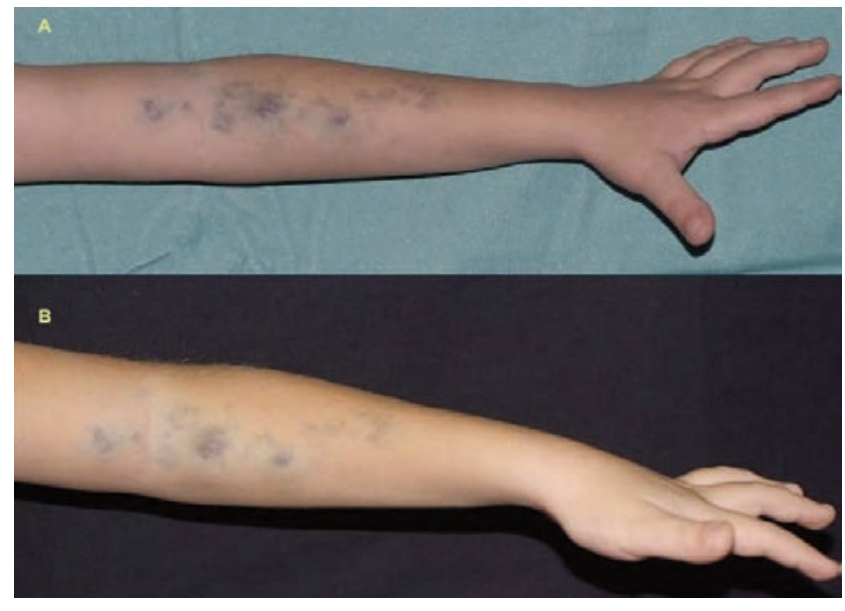

Figure 4. A) Pre-treatment clinical appearance of an arm low flow vascular malformation in a 7-year-old boy. The malformation caused great functional impairment with pain, heaviness and local sting; B) appearence after two applications of the interstitial echo-guided diode laser 980-nm ( $5 \mathrm{~W}$ for 150 sec and $10 \mathrm{~W}$ for $60 \mathrm{sec})$. A reduction of the skin component was detected but above all a marked improvement in the upper limb functional with decrease of the troublesome symptoms. 


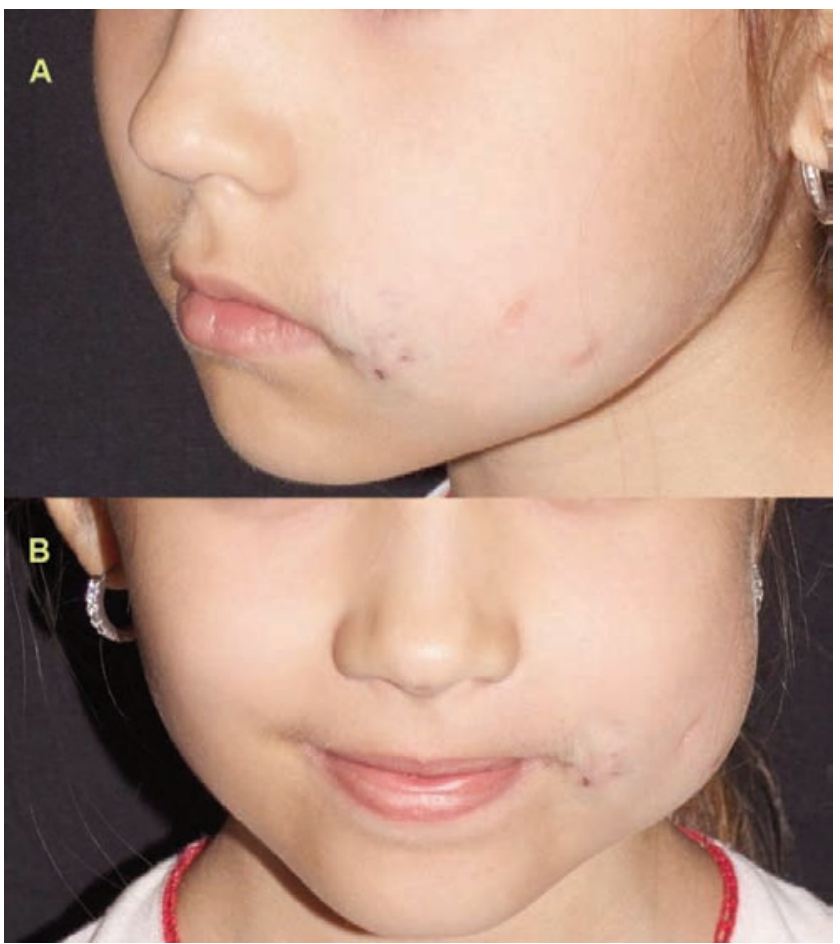

Table 1. Features considered for each patient.

\begin{tabular}{lcccccc}
\hline Patient & Age & Sex & Type & Site & Watt X seconds Treatment (n) \\
1 & 12 & M & VM & Thigh & $5 \times 120$ & 1 \\
2 & 12 & F & VM & Thorax & $10 \times 90$ & 3 \\
\hline 3 & 8 & F & VM & Thigh & 4 X120 & 3 \\
4 & 14 & F & VM & Thigh & $5 \times 120$ & 2 \\
\hline 5 & 7 & M & VM & Arm & $10 \times 90$ & 3 \\
6 & 10 & M & VM & Thigh & $5 \times 120$ & 1 \\
\hline 7 & 15 & F & VM & Thigh & $10 \times 90$ & 2 \\
8 & 5 & M & LM & Gluteus & $5 \times 120$ & 3 \\
\hline 9 & 22 & M & VM & Thorax & $5 \times 180$ & 3 \\
10 & 18 & F & VM & Thorax & $10 X 180$ & 4 \\
\hline 11 & 10 & F & VM & Cheek & $5 \times 90$ & 2 \\
12 & 11 & M & VM & Chin & $5 \times 60$ & 1 \\
\hline 13 & 18 & M & VM & Leg & $5 \times 120$ & 1 \\
14 & 10 & M & VM & Thorax & $10 \times 60$ & 2 \\
\hline 15 & 7 & M & VM & Thigh & $10 X 90$ & 1 \\
16 & 25 & M & VM & Foot & $5 \times 60$ & 1
\end{tabular}

M, male; F, female; VM, low flow venous malformation; LM, lymphatic malformation.

Figure 5. Oblique (A) and frontal (B) view of a little scar in a 10year-old patient with a cheek vascular malformation as a result of a 65 sec for $5 \mathrm{~W}$ application of interstitial echo-guided diode laser 980-nm.

rience. As already mentioned, the low-flow vascular malformations increase as the body grows and do not resolve spontaneously. They represent the most common vascular malformations present at birth, becoming symptomatic in children or young adults, with bluish skin discoloration, local swelling and pain. In these patients, before laser session have been tried, other treatments are often been accomplished unsatisfactory.

Superficial venous malformations may respond to argon, potassium titanyl phosphate (KTP) or pulsed dye laser, however the deep component is not reached and the results are moderate.

Nd:YAG laser emitting a continous wawe infrared light on a wave-lenght of $1064 \mathrm{~nm}$ creates a greater degree of thermal injury effective also on deeper lesions.

The intralesional application has been described in very few patients, however, response rates and size reduction are not exciting. ${ }^{18,19}$

Fifteen out of the 16 patients enrolled in this study affected by low flow malformations were already submitted to sclerotherapy without significant benefits. In most cases, the location was so deep in intimate relationship with nervous and muscular structure.

The chance to be surgically effective would in any case be limited by the risk of functional failures or otherwise would be forced the patient to a long period of rest.

Data collected in this study showed first of all an extreme safety and tolerability of the procedure in children and young patients, with no adverse affects such as skin atrophy, pathological scarring or hyper and hypopigmentation.

The interstitial echo guided laser allows high selectivity and more effective treatment even in areas considered critical or at high bleeding risk. In our cases a large decrease in size has been found since the first session in low-flow vascular malformations with a diameter less than $10 \mathrm{~cm}$.

All patients reported a drastic reduction of pain and swelling in the weeks following the first application. The discromic alterations of the skin presented a clear improvement as it is seen in the photos.

In lesions wider than $10 \mathrm{~cm}$, sessions were more than one but the most important decrease in symptoms was obtained since the first application. We have found instead unsatisfactory results in one patient suffering from lymphangioma, perhaps due to non-effective wave length matching lymphatic structures. ${ }^{20}$

Patients with high-flow vascular malformations were excluded, because these cases need a mandatory association of super-selective embolization before laser or surgery. This way gives up more chances to achieve lasting and satisfactory results. ${ }^{21,22}$

\section{Conclusions}

In pediatric patients low-flow vascular malformations resistant to progressive sclerotherapy or in critical anatomical sites benefit of echo guided interstitial 980 -nm diode laser.

In pediatric patients, the latter represents a less invasive approach, especially in lesions smaller than $10 \mathrm{~cm}$, providing appropriate and effective advantage in minimizing physical and psychological long-term sequelae.

\section{References}

1. Chiller KG, Passaro D, Frieden IJ Hemangiomas of infancy: clinical characteristic, morphologic subtypes, and their relationship to race, ethnicity, and sex. Arch Dermatol 2002;138:1567-76.

2. Garzon MC, Enjolras 0, Frieden IJ. Vascular tumors and vascular malformations: evidence for an association. J Am Acad Dermatol 2000;42:275-9.

3. Enjolras 0, Wassef M, Chapot R. Color 
atlas of vascular tumors and vascular malformations. Cambridge: Cambridge University Press; 2007.

4. Smolinski K, Yan AC. Hemangiomas of infancy: clinical and biological characteristics. Clin Pediatr 2005;44:747-66.

5. Enjolras 0, Soupre V, Picard A. Classification of superficial vascular anomalies. Presse Med 2010;39:457-64.

6. Wassef M, Enjolras 0. [Les malformations vasculaires superficielles: classification et histopathologie]. [Article in French]. Ann Pathol 1999;19:253-64.

7. Enjolras 0, Mulliken JB. Vascular tumors and vascular malformations (new issues). Adv Dermatol 1997;13:375-423.

8. Stücker M, Kobus S, Altmeyer P, ReichSchupke S. Review of published information on foam sclerotherapy. Dermatol Surg. 2010;36(Suppl.2):983-92.

9. Prasetyono T0, Kreshanti P. Efficacy of intra-lesional alcohol injection as alternative and/or complementary treatment of vascular malformations: a systematic review. J Plast Reconstr Aes 2010;63:10719.

10. Blaise S, Charavin-Cocuzza M, Riom H, et al. Treatment of low-flow vascular malfor- mations by ultrasound-guided sclerotherapy with polidocanol foam: 24 cases and literature review. Eur J Vasc Endovasc 2011:41:412-7.

11. Landthaler M, Hohenleutner U. Laser therapy of vascular lesions. Photodermatol Photo 2006;22:324-32.

12. Astner S, Anderson RR. Treating vascular lesions. Dermatol Ther 2005;18:267-81.

13. Poetke M, Berlien HP. Treatment of a subungual hemangioma with flashlamppumped pulsed-dye laser. J Am Acad Dermatol 2000;43:1135-6.

14. Santecchia L, Bianciardi Valassina MF, Maggiulli F, et al. Early surgical excision of giant congenital hemangiomas of the scalp in newborns: clinical indications and reconstructive aspects. J Cutan Med Surg 2013;17:106-13.

15. Cahill AM, Nijs EL. Pediatric vascular malformations: pathophysiology, diagnosis, and the role of interventional radiology. Cardiovasc Inter Rad 2011;34:691-704.

16. MacFie CC, Jeffery SL. Diagnosis of vascular skin lesions in children: an audit and review. Pediatr Dermatol 2008;25:7-12.

17. Angiero F, Benedicenti S, Benedicenti A, et al. Head and neck hemangiomas in pedi- atric patients treated with endolesional 980-nm diode laser. Photomed Laser Surg 2009;27:553-9.

18. Ulrich H, Bäumler W, Hohenleutner U, Landthaler M. Neodymium-YAG laser for hemangiomas and vascular malformations: long term results. J Dtsch Dermatol Ges. 2005;3:436-40.

19. Clymer MA, Fortune DS, Reinisch L, et al. Interstitial Nd:YAG photocoagulation for vascular malformations and hemangiomas in childhood. Arch Otolaryngol 1998;124: 431-6.

20. Lapidoth M, Ackerman L, Amitai DB, et al. Treatment of lymphangioma circumscriptum with combined radiofrequency current and $900 \mathrm{~nm}$ diode laser. Dermatol Surg 2006;32:790-4.

21. Erdmann MW, Jackson JE, Davies DM, Allison DJ. Multidisciplinary approach to the management of head and neck arteriovenous malformations. Ann Roy Coll Surg 1995;77:53-9.

22. Wu JK, Bisdorff A, Gelbert F, et al. Auricular arteriovenous malformation: evaluation, management, and outcome. Plast Reconstr Surg 2005;115:985-95. 